\title{
El ejercicio de inculturación de la espiritualidad: aporte de Gustavo Gutiérrez en "Beber en su propio pozo"
}

\author{
Orlando Solano Pinzón ${ }^{* *}$
}

Recepción: 25 de agosto de 2018 • Aprobación: 30 de septiembre de 2018

\section{Resumen}

Este artículo busca dar cuenta del carácter de inculturación de la espiritualidad que se hace presente en la obra Beber en su propio pozo, que evidencia la apropiación de los elementos propios de la cultura a través de los cuales se expresó la acción del Espíritu en las comunidades eclesiales presentes en América Latina. Este aporte puede ser fuente de inspiración para evidenciar las múltiples manifestaciones del Espíritu que pueden estarse forjando en la sociedad y ser referente importante ante la necesidad de inculturar tanto la teología como la espiritualidad hoy.

Palabras clave: comunidades eclesiales, espíritu, espiritualidad de la liberación, inculturación, Gustavo Gutiérrez.

\footnotetext{
Artículo de investigación, vinculado con el proyecto “La inculturación de la teología como hermenéutica de la apropiación: el De vita Moysis de Gregorio de Nisa, ejemplo de inculturación", desarrollado en la Pontificia Universidad Javeriana, fue presentado en el Primer Congreso Internacional de Teología Latinoamericana y del Caribe: 50 años de Medellín: Iglesia y signos de los tiempos, desarrollado en la Universidad Santo Tomás entre el 16 y el 19 de octubre de 2018. Citar como: Solano Pinzón, O. (2018). El ejercicio de inculturación de la espiritualidad: aporte de Gustavo Gutiérrez en "Beber en su propio pozo". Albertus Magnus, IX(2), 59-70 Doi: https://doi.org/10.153322/5005413.5100.

** Pontificia Universidad Javeriana. Bogotá, Colombia. Orcid: http://orcid.org/0000-00034446-626X. Correo electrónico: o.solano@javeriana.edu.co
} 


\title{
The exercise of inculturation of spirituality: Contribution of Gustavo Gutiérrez in "Drinking from your own well”
}

\begin{abstract}
This paper seeks to give an account of the inculturation character of spirituality that is present in the work: "Drinking from your own well", evidencing the appropriation of the elements of culture through which the action of the Spirit was expressed in the ecclesial communities existing in Latin America. This contribution can be a source of inspiration to account for the multiple manifestations of the Spirit that can be forged within society and be an important reference to the need to inculturate both theology and spirituality today.

Keywords: ecclesial communities, spirit, spirituality of liberation, inculturation, Gustavo Gutiérrez.

\section{O exercício da inculturação da espiritualidade: contribuição de Gustavo Gutiérrez em "Beber em seu próprio bem"}

\section{Resumo}

A presente redação busca dar conta do caráter de inculturação da espiritualidade presente na obra: "Beber em seu próprio bem", evidenciando a apropriação dos elementos da cultura através dos quais se manifestou a ação do Espírito nas comunidades eclesiais presentes na América Latina. Essa contribuição pode ser uma fonte de inspiração para explicar as múltiplas manifestações do Espírito que podem ser forjadas dentro da sociedade e ser uma referência importante para a necessidade de inculturar teologia e espiritualidade hoje.

Palavras-chave: comunidades eclesiais espírito, espiritualidade de libertação, inculturação, Gustavo Gutiérrez. 


\section{Introducción}

Como bien señala Robira (1996), "una cultura solo puede ser fecundada desde dentro, desde su libertad, no desde fuera y desde el dominio exterior ejercido con medios puramente humanos" (p. 327). Tal proceso de fecundación es aquello que está a la base del significado de la categoría inculturación, que desde hace varias décadas hizo su incursión en el lenguaje teológico y eclesiástico, pero que a la fecha son pocos los ejemplos que pueden dar cuenta de su praxis.

Dentro de los escasos ejemplos del adecuado ejercicio de inculturación de la teología en América Latina, la intensión de este artículo se centra en ofrecer algunos elementos fundamentales del trabajo de inculturación realizado por Gustavo Gutiérrez (1983) en su obra Beber en su propio pozo. Para tal efecto, en un primer momento, abordaremos la comprensión de la categoría inculturación, posteriormente haremos una breve descripción del personaje y la obra objeto de estudio y, por último, explicitaremos los elementos de inculturación que están presentes en dicha obra.

Es importante tener presente que la obra en cuestión constituye una síntesis de teología espiritual, que Gustavo Gutiérrez logró sistematizar a partir de la experiencia vital y el contacto directo con comunidades eclesiales de base de América Latina. Dicha obra no es solo expresión de la unión entre espiritualidad y teología, disuelta durante muchos años en la historia de la teología, sino que permite evidenciar un trabajo de gran sensibilidad espiritual para dar cuenta de la novedad del Espíritu en medio de la realidad social latinoamericana y lograr inculturar dicha novedad a partir de elementos constitutivos de la cultura popular en la cual emerge.

\section{Noción de inculturación}

En el sentido original del concepto inculturación que, según Andrés Tornos (2001), fue creado por Melville Herskovits, se buscaba expresar el esfuerzo del individuo por impregnarse de todo lo implicado en la cultura buscando garantizar con ello una convivencia sana con sus semejantes. Dicho concepto, al pasar al lenguaje teológico, ${ }^{1}$ fue asociado con la palabra e idea de Encarnación, lo que permitió

1 Para mayor información sobre los antecedentes que llevaron al uso de la categoría inculturación en el ámbito eclesial y teológico, puede ser útil el trabajo realizado por Acosta (2001, pp. 29-91). 
afirmar que los Padres de la Iglesia son maestros de inculturación en el sentido de repensarlo en el ambiente cultural grecorromano. Lo anterior debido a que la Encarnación está en el centro de su pensamiento, de su vida de fe, de su labor pastoral y de su trabajo escriturístico (Poupard, 1995).

Esa asociación de la inculturación con la Encarnación se hizo pública en el ámbito eclesiástico durante un sínodo de catequesis llevado a cabo en 1977, en una intervención realizada por el padre Pedro Arrupe, quien asumió explícitamente la categoría inculturación en clave de Encarnación (Solano, 2015). Al respecto, conviene indicar, como afirma Suess (1990), al hablar de las dos categorías en cuestión, que

entre el significado de la inculturación y el de la encarnación no hay una identidad, pero sí una relación de analogía entre los dos términos. Y esta analogía se muestra en la articulación dialéctica de dos niveles: a) en la superación de la distancia y diferencia que impiden la comunicación y la solidaridad y, por tanto, la proximidad amable y crítica y la igualdad solidaria; b) en el respeto a la alteridad, en la no identificación con el otro. (pp. 411-412)

Comprender la inculturación en clave de Encarnación enriquece sobremanera su significado y le confiere un carácter liberador y redentor, debido a que la Encarnación es liberadora y salvífica (De França, 2004). En esta breve descripción del concepto, es de gran importancia la referencia a la apropiación de la matriz cultural en el proceso de inculturación debido a que, según Padovese (1996), la inculturación es un hecho histórico que solo se verifica entre personas y debe estar abierta a todo el universo simbólico de la diversidad de pueblos con los que entra en contacto la fe.

\subsection{Inculturación de la espiritualidad}

Una vez abordada la categoría inculturación, conviene evocar la comprensión que Gutiérrez tiene sobre la espiritualidad y explicitar la particularidad que asume el hablar de inculturación de la espiritualidad. Para nuestro autor en cuestión, la espiritualidad es el dominio del Espíritu, es una forma concreta movida por el Espíritu de vivir el Evangelio. “Una manera precisa de vivir 'ante el Señor' en solidaridad con todos los hombres, 'con el Señor' y ante los hombres" (1972, p. 267).

Ahora bien, cuando hablamos de inculturación de la espiritualidad, estamos haciendo referencia al proceso de apropiación de una matriz cultural, que en el caso de Gutiérrez es la cultura popular presente en los pueblos latinoamericanos, 
para vivir, repensar, decir y celebrar desde ella la manera como se desoculta Dios en los acontecimientos de la historia que viven las mujeres y los hombres que comparten dicha matriz. Si bien el lenguaje a través del cual se recibe y expresa el desocultarse de Dios siempre se queda corto, pueden, de manera analógica, hacerse acercamientos provisorios, debido a que el lenguaje humano está abierto a hacer presente y comunicar una realidad que lo trasciende (Codina, 1997).

\section{Gustavo Gutiérrez y la síntesis espiritual}

Gustavo Gutiérrez nació en Lima el 8 de junio de 1928, y como muchos otros teólogos latinoamericanos contemporáneos, no puede comprenderse sin la unión entre su itinerario vital y su ejercicio intelectual. Su ser de teólogo refleja la persona que es y la realidad social y humana que le circunda. Por ello, ha afirmado: "Se cree en Dios a partir de una situación histórica determinada; el creyente forma parte [...] de un tejido cultural y social", luego, "se intenta pensar esa fe" (Gutiérrez, 1989, p. 18).

El hecho de provenir de una familia indígena le permitió conocer de primera mano el dolor indebido de los pobres que están condenados a morir antes de tiempo. Además, su experiencia infantil y juvenil que le pone desde muy pronto en contacto con el dolor que experimenta a causa de sus quebrantos de salud provoca en él una sintonía particular con el mundo y el entorno del sufrimiento, el padecimiento de tantos seres humanos en situación de pobreza. Esta particular disposición permite comprender mejor los antecedentes del ejercicio de inculturación que plasmará en su obra Beber en su propio pozo.

Unido a la experiencia personal de cercanía a la realidad de los pobres, desde la que realizó el hallazgo del Dios del Evangelio, es importante hacer referencia a la excelente formación teológica recibida en Europa, que aporta a la consolidación de su estilo particular de vivir, de pensar y de escribir. Refiriéndose a su formación teológica, él mismo afirma:

El estudio de la primera cuestión de la Suma de santo Tomás, el aporte de Melchor Cano sobre los lugares teológicos, el clásico libro de Gardeil sobre estos asuntos me apasionaron. Devoré en unas vacaciones el artículo "Teología" de Y. Congar en el Diccionario de teología católica; su perspectiva histórica me sacó de un modo casi exclusivamente racional de enfocar el estudio teológico, abriéndome a otras orientaciones (la Escuela de Tubinga, por ejemplo). Más tarde la lectura de un libro, de discreta circulación, de M.-D. Chenu, La escuela de Le Saulchoir, me 
descubrió el alcance de la historia humana y la vida misma de la Iglesia como un lugar teológico [...] Este interés hizo que en los tratados de teología que estudié estuviese muy atento al aspecto metodológico y a la relación de la teología con las fuentes de la Revelación. A ello contribuyó de manera particular la insistencia de muchos de mis profesores de Lyon en la Sagrada Escritura. (Gutiérrez, 2003, p. 7)

La estrecha relación entre su itinerario vital, su experiencia espiritual y su producción intelectual dan buena cuenta de los elementos que se implican en un proceso de inculturación. Además, los aspectos biográficos van a permitir comprender mejor el esfuerzo por encarnar la acción del Espíritu y dar cuenta de dicha acción a partir de las experiencias más significativas propias de un ambiente cultural popular, que serán sistematizadas en la obra a la cual nos referiremos a continuación.

\section{1. "Beber en su propio pozo"}

Beber en su propio pozo es expresión de una profunda experiencia espiritual, por esta razón afirma Gutiérrez (1983) que “en toda gran espiritualidad tenemos al inicio un nivel de experiencia. Luego una reflexión sobre esa vivencia, lo que permite proponerla al conjunto de la comunidad cristiana como un modo de seguir a Cristo" (p. 83). En este sentido, para Gutiérrez, la reflexión teológica de una experiencia implica asumirla "confrontándola con la palabra del Señor, con el pensamiento de su tiempo, así como con otras maneras de entender el seguimiento" (p. 84).

Debido a que esta obra busca recoger la experiencia espiritual que está a la base de la experiencia de las comunidades eclesiales comprometidas en el proceso de liberación, es oportuno afirmar que dicha experiencia en América Latina está mediada por las distintas formas de presencia de Dios que, según Gutiérrez (1972), "condicionan las formas de nuestro encuentro con él". Por tanto, "si la humanidad, si cada hombre es el templo vivo de Dios, a Dios lo encontramos en el encuentro con los hombres, en el compromiso con el devenir histórico de la humanidad" (pp. 250-251).

Ahora bien, si "toda gran espiritualidad está ligada a los grandes movimientos históricos de su época" (Gutiérrez, 1983, p. 45), la espiritualidad de la liberación tiene como contexto de gestación los movimientos de liberación presentes en las diferentes latitudes del continente, que en medio de las condiciones de inhumana pobreza y explotación se van haciendo conscientes de su condición 
de explotación y sometimiento, y aspiran a la liberación de todo aquello que limita el ejercicio de su libertad e impide realizarse a sí mismos como humanos.

Esta experiencia espiritual de encuentro con Dios en el encuentro con los pobres y su anhelo de liberación constituye el objeto del cual busca dar razón Gutiérrez en la obra en cuestión. La novedad de la experiencia espiritual permitió comprender que el seguimiento de Jesús se presenta, no a través de una ruta individual, sino en una aventura comunitaria y en solidaridad con los pobres y sus anhelos de liberación.

La afirmación fundamental que está a la base de toda la obra en cuestión es que en América Latina existe un pozo con agua de vida, que lo han ido llenando con su fe, con su esperanza, con su compromiso y con su gozo, con sus lágrimas y con su sangre muchas veces los cristianos del pueblo pobre que se han comprometido con la liberación. Porque existe este pozo de vida cristiana puede haber espiritualidad; porque esa vida cristiana es novedosa puede haber una nueva espiritualidad.

\section{3. "Beber en su propio pozo" como experiencia de inculturación de la espiritualidad}

En esta forma de ser fiel al Señor dentro del proceso de liberación, serán las experiencias más significativas vividas en el pueblo pobre las que van a configurar su particular espiritualidad. Los rasgos de la espiritualidad que de la experiencia de las comunidades eclesiales surgen están íntimamente correlacionados en cuanto configuran un modo particular de apropiar la existencia, de inculturar la acción del Espíritu en la cultura popular dentro del proceso de liberación. A continuación, abordaremos brevemente cada uno de ellos.

\subsection{Conversión}

La conversión ha sido siempre la piedra de toque de toda espiritualidad, pero dentro del proceso de liberación adquiere una particularidad, ya que dicha conversión surge del encuentro con Cristo presente en los pobres y se constituye en el centro integrador de la espiritualidad de la liberación. Gutiérrez (1972) la concibe como "una transformación radical de nosotros mismos y un pensar, sentir y vivir como Cristo presente en el hombre despojado y alienado" (p. 268). En este sentido, la conversión implica la exigencia de un estilo de vida que frente a la realidad que se vive en América Latina involucra una acción comprometida y solidaria con el proceso de liberación de los pobres y explotados, que incluye la 
transformación del medio socioeconómico, político, cultural y humano en que se encuentran. Además, dicha conversión requiere una revisión de las propias estructuras de la Iglesia y de la vida de sus miembros (Gutiérrez, 1979).

La conversión se vive, no como un hecho puntual, sino como un proceso permanente en medio de los conflictos que implican rupturas referidas a categorías mentales y a la forma de relacionarnos con los demás, de afrontar la vida y de encarnar la fe. En fin, ruptura "con todo aquello que trabe una solidaridad real y profunda con aquellos que sufren, en primer lugar, una situación de miseria e injusticia" (Gutiérrez, 1972, p. 269).

\subsection{Gratuidad}

La espiritualidad de la liberación está impregnada de una vivencia de gratuidad, que constituye el terreno de la libertad, del amor, de la contemplación, de la oración y la donación ilimitada, que supone el señorío y la responsabilidad ante la historia (Gutiérrez, 1972). La vivencia de la gratuidad no es una evasión, sino el clima en que baña una eficacia histórica buscada cada vez más ardorosamente por aquello mismo que nos revela la gratuidad: el amor preferencial de Dios por el pobre. En este sentido, vivir en el Espíritu es vivir en el amor.

Dicha gratuidad estimula al compromiso en la búsqueda de resultados basados en cálculos y el conocimiento relacional y causal de la realidad, que explicita la capacidad de amar como Dios ama. Pero simultáneamente crece la vivencia de la gratuidad, no como un refugio ante la impotencia histórica, sino como una exigencia de compromiso real y eficaz. Al respecto, Gutiérrez (1972) afirmará que el compromiso con el proceso de liberación de los pobres y explotados debe ser lúcido, realista y concreto, más aún, "no solo con generosidad, sino también con análisis de situación y con estrategia de acción" (p. 268).

\subsection{Alegría}

La alegría como rasgo de la espiritualidad de la liberación está ligada a las raíces de la cultura latinoamericana en tanto pueblo festivo. El talante cristiano de esta experiencia de alegría y, por ende, su sentido liberador "nace del don ya recibido y todavía esperado, que se expresa en el presente, pese a la dureza y a las tensiones de la lucha por la construcción de una sociedad justa" (Gutiérrez, 1972, p. 271). Este talante de esperanza es vivido en el pueblo en la alegría, que no es fácil, pero es real. No es la alegría superficial de la inconsciencia o la resignación, 
sino aquella que nace de la esperanza de que el maltrato y el sufrimiento serán vencidos.

Es oportuno cerrar este rasgo de la espiritualidad con un testimonio que evoca Gutiérrez (1983) de las comunidades cristianas de El Quinché:

En los días de noche buena, año nuevo y otras fiestas, han venido hasta acá algunos padres y han hecho misa en varios pueblos. Esto nos da mucha alegría pero también estamos con pena porque los pueden matar. Nosotros deseamos que ellos vengan, que venga monseñor Gerardi, los padres y las hermanas, pero no ahora. Queremos que sigan vivos, que se cuiden para que puedan seguir ayudando al pueblo ahora y después así como lo han hecho siempre. (p. 153)

\subsection{Pobreza espiritual}

La pobreza espiritual es comprendida por Gutiérrez (1972) como la total disponibilidad ante el Señor, la cual define la postura total de la existencia humana frente a Dios, los hombres y las cosas (desde una actitud de libertad ante ellas). Más aún, podría comprenderse como una actitud vital, global y sintética, que dinamiza la totalidad y el detalle de nuestra vida.

Ahora bien, dicha pobreza se encarna en el compromiso de hacerse solidario frente a las situaciones que afectan la vida de los demás, pues, solo si hay una apertura y disponibilidad, es posible comprometerse con los otros. Este talante de compromiso que surge de la comprensión de la pobreza espiritual es tan fuerte en la espiritualidad de la liberación que Gutiérrez (1972) llega a afirmar que "solo rechazando la pobreza y haciéndose pobre para protestar contra ella, podrá la Iglesia predicar algo que le es propio: la 'pobreza espiritual'; es decir, la apertura del hombre y de la historia al futuro prometido por Dios" (p. 385).

\subsection{Comunidad}

La experiencia espiritual que emerge en América Latina es una experiencia comunitaria que se concreta en las diferentes comunidades eclesiales que se van convirtiendo en focos de evangelización y en motores de liberación y desarrollo. Según Gutiérrez (1972), “Dios convoca, y se encarna en la comunidad de fe que se entrega al servicio de todos los hombres" (p. 30). Sin embargo, la comunidad se hace viable cuando se da la experiencia de soledad que hace posible la comunión y genera 
comunidad como encuentro profundo con Dios, que constituye la dimensión mística de toda espiritualidad.

En el seno de las comunidades eclesiales, se gesta una manera de ser fiel a la acción del Espíritu, una espiritualidad que se enriquece con las experiencias de compromiso existencial en los caminos concretos de promoción liberadora. Además, se consolida la vocación teológica en tanto se suscita y se ejerce en el seno de la comunidad. Al respecto, afirma Gutiérrez (2003) desde su propia experiencia:

La tarea del teólogo consiste entonces en aportar a la comunidad lo que un entrenamiento académico le haya podido dar, como un mejor conocimiento y familiaridad con la Sagrada Escritura, la tradición y la enseñanza eclesial, la teología contemporánea. La teología no es una tarea individual, sino una función eclesial. Ella se hace desde la palabra de Dios recibida y vivida en la Iglesia, en orden a su anuncio a toda persona humana y en especial a los desheredados de este mundo [...] Contrariamente a lo que muchos piensan, la experiencia muestra que la cercanía a las comunidades eclesiales de base obliga a un gran rigor en el quehacer teológico. (p. 25)

El talante comunitario que hace parte de la tarea del teólogo fue determinante en la experiencia de Gutiérrez, no solo porque le permitió encarnar mejor su praxis de fe, sino porque le posibilitó corroborar que la vida en comunidad es posible por y hace evidente la acción del Espíritu en tanto "solo en comunidad podemos escuchar, acoger y anunciar el don y la gracia del Señor, el llamado privilegiado a superar todo lo que rompa la comunión fraterna [...] La comunidad es también instancia donde se hace memoria de la muerte y resurrección del Señor" (Gutiérrez, 1983, p. 172).

\section{Conclusión}

A manera de conclusión, es posible afirmar que la teología espiritual de Gustavo Gutiérrez es un ejercicio de inculturación que no se explica ni se comprende sino desde una espiritualidad profundamente encarnada en las circunstancias de la historia. En este sentido, se comprende mejor la expresión "nuestra metodología es nuestra espiritualidad y nuestra espiritualidad es nuestra forma de vida" (Gutiérrez, 2003, p. 108). En efecto, no puede haber fracturas, ni distanciamientos, ni vacíos, ni separaciones entre la praxis de fe del teólogo, su estilo de vida y su esfuerzo por hacer inteligible dicha fe a todos sus interlocutores. 
La inculturación no es una apuesta novedosa de hacer teología espiritual, sino la apuesta hermenéutica que debe estar presente en toda teología, para que no redunde en discursos ilegibles, desentendidos de los problemas reales que viven las comunidades eclesiales, que constituyen el lugar eclesial del quehacer teológico. La realidad que vivimos hoy exige el ejercicio continuo de inculturación del Evangelio, de la fe y de la teología, para que las culturas sean fecundadas desde dentro y la fe no sea un elemento decorativo en la vida de las personas sino fuente inagotable de vida.

Para terminar, es posible afirmar que la teología de Gustavo Gutiérrez no se explica ni se comprende sino desde una espiritualidad encarnada, y por ello profundamente arraigada en las circunstancias de la historia, que en el caso de América Latina estuvo y sigue marcada por la pobreza y la opresión. Su trabajo de inculturación sigue siendo vigente y se convierte en fuente de inspiración para poder dar cuenta de la variedad de formas a través de la cuales el Espíritu sigue generando vida en medio de las realidades de violencia, dominación y muerte anticipada.

\section{Referencias}

Acosta Nassar, R. J. (2001). La inculturación en los trabajos de las Conferencias Generales del Episcopado Latinoamericano en Puebla (1979) y en Santo Domingo (1992). Roma, Italia: Pontificia Universitas Sanctae Crucis.

Codina, V. (1997). Los caminos del Oriente cristiano: iniciación a la teología oriental. Santander, España: Sal Terrae.

De França Miranda, M. (2004). La inculturación de la fe: un abordaje teológico. Bogotá, Colombia: Consejo Episcopal Latinoamericano.

Gutiérrez, G. (1972). Teología de la liberación. Salamanca, España: Sígueme.

Gutiérrez, G. (1979). Pobres y liberación en Puebla. Páginas, 4, 1-32.

Gutiérrez, G. (1983). Beber en su propio pozo: en el itinerario espiritual de un pueblo. Lima, Perú: CEP.

Gutiérrez, G. (1989). El Dios de la vida. Lima, Perú: Instituto Fray Bartolomé de Las Casas.

Gutiérrez, G. (2003). Densidad del presente. Salamanca, España: Sígueme.

Padovese, L. (1996). Introducción a la teología patrística. Estella, España: Verbo Divino.

Poupard, P. C. (1995). Los Padres de la Iglesia: actualidad de una inculturación de la fe. En D. Ramos-Lissón, M. Merino y A. Viciano (Eds.), Diálogo fe-cultura en la antigüedad cristiana (pp. 27-46). Pamplona, España: Eunate. 
Rovira Belloso, J. M. (1996). Introducción a la teología. Madrid, España: Biblioteca de Autores Cristianos.

Solano Pinzón, O. (2015). Inculturación de la teología en Gregorio Nisa: antecedentes. Theologica Xaveriana, 65(179), 157-183. DOI: 10.11144/javeriana.tx65-179.itgn

Suess, P. (1990). Inculturación. En I. Ellacuría y J. Sobrino, J. Mysterium liberationis: conceptos fundamentales de la teología de la liberación (pp. 411-412). Madrid, España: Trotta.

Tornos, A. (2001). Inculturación teología y método. Madrid, España: Universidad de Comillas. 